\title{
Experimental quantum teleportation of a two-qubit composite system
}

\author{
QIANG ZHANG ${ }^{1}{ }^{2 *}$, ALEXANDER GOEBEL ${ }^{1}$, CLAUDIA WAGENKNECHT ${ }^{1}$, YU-A0 CHEN ${ }^{1}$, BO ZHA0 ${ }^{1}$, \\ TAO YANG ${ }^{2}$, ALOIS MAIR ${ }^{1}$, JÖRG SCHMIEDMAYER ${ }^{1,3}$ AND JIAN-WEI PAN ${ }^{1,2 *}$
}

\author{
${ }^{1}$ Physikalisches Institut, Universität Heidelberg, Philosophenweg 12, D-69120 Heidelberg, Germany \\ ${ }^{2}$ Hefei National Laboratory for Physical Sciences at Microscale and Department of Modern Physics, University of Science and Technology of China, Hefei, Anhui 230027, \\ People's Republic of China \\ ${ }^{3}$ Atominstitut der Österreichischen Universitäten, TU-Wien, A-1020 Vienna, Austria \\ *e-mail: bowzhang@mail.ustc.edu.cn; jian-wei.pan@physi.uni-heidelberg.de
}

Q uantum teleportation ${ }^{1}$, a way to transfer the state of a quantum system from one location to another, is central to quantum communication ${ }^{2}$ and plays an important role in a number of quantum computation protocols ${ }^{3-5}$. Previous experimental demonstrations have been implemented with single photonic ${ }^{6-11}$ or ionic qubits ${ }^{12,13}$. However, teleportation of single qubits is insufficient for a large-scale realization of quantum communication and computation ${ }^{2-5}$. Here, we present the experimental realization of quantum teleportation of a twoqubit composite system. In the experiment, we develop and exploit a six-photon interferometer to teleport an arbitrary polarization state of two photons. The observed teleportation fidelities for different initial states are all well beyond the state estimation limit of 0.40 for a two-qubit system ${ }^{14}$. Not only does our six-photon interferometer provide an important step towards teleportation of a complex system, it will also enable future experimental investigations on a number of fundamental quantum communication and computation protocols $s^{3,15-18}$.

Our experimental scheme $\mathrm{e}^{19,20}$ closely follows the original proposal for teleportation of single qubits ${ }^{1}$. In the two-qubit teleportation, the sender, Alice, wants to send an unknown state of a system composed of qubits 1 and 2,

$$
|\chi\rangle_{12}=\alpha|H\rangle_{1}|H\rangle_{2}+\beta|H\rangle_{1}|V\rangle_{2}+\gamma|V\rangle_{1}|H\rangle_{2}+\delta|V\rangle_{1}|V\rangle_{2},
$$

to a distant receiver, Bob (Fig. 1). Here we discuss our protocol in terms of polarization photonic qubits. $|H\rangle$ and $|V\rangle$ denote horizontal and vertical linear polarizations respectively, and $\alpha, \beta, \gamma$ and $\delta$ are four arbitrary complex numbers satisfying $|\alpha|^{2}+|\beta|^{2}+$ $|\gamma|^{2}+|\delta|^{2}=1$. To achieve teleportation, Alice and Bob first have to share two ancillary entangled photon pairs (photon pairs 3-5 and 4-6), which are prepared in the Bell state $\left|\Phi^{+}\right\rangle=(|H\rangle|H\rangle+$ $|V\rangle|V\rangle) / \sqrt{2}$. The two-qubit teleportation scheme then works as follows.

Alice first teleports the state of photon 1 to photon 5 following the standard teleportation protocol. In terms of the four Bell states of photons 1 and 3, $\left|\Phi^{ \pm}\right\rangle_{13}=\left(|H\rangle_{1}|H\rangle_{3} \pm|V\rangle_{1}|V\rangle_{3}\right) / \sqrt{2}$ and $\left|\Psi^{ \pm}\right\rangle_{13}=\left(|H\rangle_{1}|V\rangle_{3} \pm|V\rangle_{1}|H\rangle_{3}\right) / \sqrt{2}$, the combined state of photons 1, 2, 3 and 5 can be rewritten as

$$
\begin{aligned}
|\chi\rangle_{12}\left|\Phi^{+}\right\rangle_{35}= & \frac{1}{2}\left(\left|\Phi^{+}\right\rangle_{13}|\chi\rangle_{52}+\left|\Phi^{-}\right\rangle_{13} \hat{\sigma}_{5 z}|\chi\rangle_{52}+\left|\Psi^{+}\right\rangle_{13} \hat{\sigma}_{5 x}|\chi\rangle_{52}\right. \\
& \left.-\left|\Psi^{-}\right\rangle_{13} i \hat{\sigma}_{5 y}|\chi\rangle_{52}\right)
\end{aligned}
$$

where $\hat{\sigma}_{x}, \hat{\sigma}_{y}$ and $\hat{\sigma}_{z}$ are the usual Pauli operators. Equation (1) implies that by carrying out a joint Bell-state measurement (BSM) on qubits 1 and 3, Alice projects the state of qubits 5 and 2 onto one of the four corresponding states. After she has told Bob her BSM result via a classical communication channel, Bob can convert the state of qubits 5 and 2 into the original state $|\chi\rangle_{52}$ by applying to photon 5 a corresponding local unitary transformation $\left(\hat{I}, \hat{\sigma}_{x}, \hat{\sigma}_{y}, \hat{\sigma}_{z}\right)$, independent of the original state.

Similarly, the combined state of photons 2, 4, 5 and 6 can be rewritten in terms of the four Bell states of photons 2 and 4 as

$$
\begin{aligned}
|\chi\rangle_{52}\left|\Phi^{+}\right\rangle_{46}= & \frac{1}{2}\left(\left|\Phi^{+}\right\rangle_{24}|\chi\rangle_{56}+\left|\Phi^{-}\right\rangle_{24} \hat{\sigma}_{6 z}|\chi\rangle_{56}+\left|\Psi^{+}\right\rangle_{24} \hat{\sigma}_{6 x}|\chi\rangle_{56}\right. \\
& \left.-\left|\Psi^{-}\right\rangle_{24} i \hat{\sigma}_{6 y}|\chi\rangle_{56}\right) .
\end{aligned}
$$

Following the above procedure, Alice can also teleport the state of photon 2 to photon 6. First, Alice carries out a joint BSM on photons 2 and 4 and sends the BSM result to Bob. On receiving the BSM result, by applying to photon 6 a corresponding local unitary transformation $\left(\hat{I}, \hat{\sigma}_{x}, \hat{\sigma}_{y}, \hat{\sigma}_{z}\right)$, Bob can convert the state of qubits 5 and 6 into the original state

$$
|\chi\rangle_{56}=\alpha|H\rangle_{5}|H\rangle_{6}+\beta|H\rangle_{5}|V\rangle_{6}+\gamma|V\rangle_{5}|H\rangle_{6}+\delta|V\rangle_{5}|V\rangle_{6}
$$

to accomplish the task of the most general two-qubit teleportation.

The above scheme has a remarkable feature: it teleports the two photonic qubits, 1 and 2, individually. This way, neither the two original qubits nor the teleported qubits have to be in the same place. Such a flexibility is desired in distributed quantum 


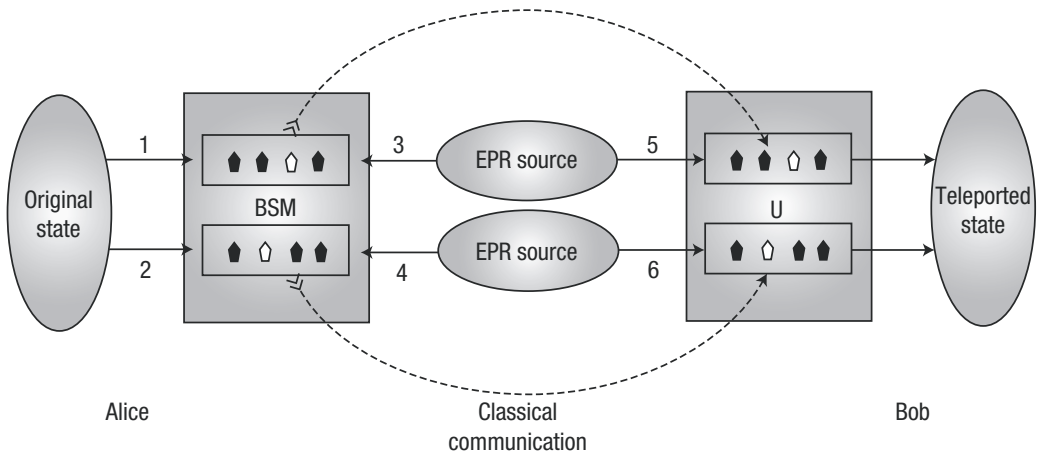

Figure 1 Schematic diagram showing the principle of two-qubit quantum teleportation. Alice wants to teleport an unknown state of a system composed of photons 1 and 2 to Bob. To do so, Alice and Bob first share two entangled photon pairs (EPR source), photon pairs 3-5 and 4-6. Alice then carries out a joint Bell-state measurement (BSM) both on photons 1 and 3 and on photons 2 and 4, respectively. On receiving Alice's BSM results via classical communication, Bob can then carry out a corresponding unitary transformation $(U)$ on both photons 5 and 6 to covert them into the original state of photons 1 and 2.

information processing, such as quantum telecomputation ${ }^{5}$ and quantum secret sharing ${ }^{21}$. Moreover, the method of teleporting each qubit of a composite system individually can be easily generalized to teleport an $N$-qubit complex system.

Although significant experimental advances have been achieved in teleportation of single (photonic and ionic) qubits, the realization of teleportation of a composite system containing two or more qubits has remained a real experimental challenge. This is because, on one hand, the recent photonic experiments ${ }^{9-11}$ had a too low six-photon coincidence rate. On the other hand, the experiments with trapped ions ${ }^{12,13}$ are limited by the finite lifetime of ion qubits due to decoherence and the non-ideal fidelity of quantum logic operation between ion qubits. As photons are robust against decoherence and high-precision unitary transformations for photons can be carried out with linear optical devices, in the present experiment we still chose to use polarization-entangled photon pairs via parametric down-conversion ${ }^{22}$ as the main resource. Various efforts have been made to greatly improve the brightness and stability of the entangled photon sources.

A schematic diagram of our experimental setup is shown in Fig. 2. A high-intensity ultraviolet laser (see the Supplementary Information) successively passes through two $\beta$-barium borate (BBO) crystals to generate three polarization-entangled photon pairs ${ }^{22}$. The ultraviolet laser with a central wavelength of $390 \mathrm{~nm}$ has a pulse duration of $180 \mathrm{fs}$, a repetition rate of $76 \mathrm{MHz}$ and an average power of $1.0 \mathrm{~W}$. All three photon pairs are originally prepared in the Bell state $\left|\Phi^{+}\right\rangle=(|H\rangle|H\rangle+|V\rangle|V\rangle) / \sqrt{2}$. Besides the high-intensity ultraviolet laser, significant efforts have been made to achieve better collection efficiency and stability of the entangled photon sources (see the Supplementary Information). Following these efforts, we managed to observe on average $10^{5}$ photon pairs per second from each source. This is almost five times brighter than the source achieved in a recent teleportation experiment ${ }^{11}$. With this high-intensity entangled photon source we could obtain in total 10 six-photon events per minute. This is two orders of magnitude higher than any former photonic teleportation experiments could have achieved.

With the help of wave plates and polarizers, we prepared photon pair 1-2 in the desired two-qubit state $|\chi\rangle_{12}$ that is to be teleported. Photon pairs 3-5 and 4-6, which are in the state $\left|\Phi^{+}\right\rangle$, are used as the two ancillary pairs.

To implement two-qubit teleportation, it is necessary to carry out a joint BSM on photons 1 and 3 and photons 2 and 4, respectively. To demonstrate the working principle of two-qubit teleportation, it is sufficient to identify one of the four Bell states in both BSMs, although this will result in a reduced efficiencythe fraction of success - of $1 / 16$. In the experiment, we decide to analyse the Bell state $\left|\Phi^{+}\right\rangle$. This is achieved by interfering photons 1 and 3 and photons 2 and 4 on a polarizing beam splitter (PBS13 and PBS24) (23 $^{2}$. To interfere photons 1 and 3 (photons 2 and 4) on the PBS13 (PBS24), it has to be guaranteed that the two photons have good spatial and temporal overlap at the PBS such that they are indistinguishable. To achieve this, the two outputs of the PBSs are spectrally filtered $\left(\Delta \lambda_{\text {FWHM }}=2.8 \mathrm{~nm}\right.$ ) and monitored by fibrecoupled single-photon detectors ${ }^{24}$. Moreover, perfect temporal overlap is accomplished by adjusting the path length of photon 3 (photon 2) by a delay prism 1 (prism 2) to observe 'HongOu-Mandel'-type interference fringes behind the PBS13 (PBS24) in the \pm basis $^{23}$, where $| \pm\rangle=(|H\rangle \pm|V\rangle) / \sqrt{2}$. With the help of polarizers oriented at $45^{\circ}$, the required projection of photons 1 and 3 ( 2 and 4$)$ onto $\left|\Phi^{+}\right\rangle$can then be achieved by detecting behind PBS13 (PBS24) a $|+\rangle|+\rangle$ or $|-\rangle|-\rangle$ coincidence between detectors D1 and D3 (D2 and D4) ${ }^{23}$. Note that, in the experiment, only the $|+\rangle|+\rangle$ coincidence is registered, which further reduces the teleportation efficiency to $1 / 64$. However, by inserting one PBS and two detectors behind each output of PBS13 and PBS24, respectively, both $\left|\Phi^{+}\right\rangle$(by detecting a $|+\rangle|+\rangle$or $|-\rangle|-\rangle$coincidence) and $\left|\Phi^{-}\right\rangle$(by detecting either a $|+\rangle|-\rangle$or a $|-\rangle|+\rangle$coincidence) can be identified and thus the efficiency can be increased up to $1 / 4$ (ref. 25).

As shown in equations (1) and (2), the projection measurements onto $\left|\Phi^{+}\right\rangle_{13}$ and $\left|\Phi^{+}\right\rangle_{24}$ leave photons 5 and 6 in the state $|\chi\rangle_{56}$, that is, the original state of photons 1 and 2. To demonstrate that our two-qubit teleportation protocol works for a general unknown polarization state of photons 1 and 2, we decide to teleport three different initial states: $|\chi\rangle_{\mathrm{A}}=|H\rangle|V\rangle,|\chi\rangle_{\mathrm{B}}=(|H\rangle+|V\rangle)(|H\rangle-i|V\rangle) / \sqrt{2}$ and $|\chi\rangle_{\mathrm{C}}=(|H\rangle|V\rangle-|V\rangle|H\rangle) / \sqrt{2}$. $|\chi\rangle_{\mathrm{A}}$ is simply one of the four computational basis vectors in the two-qubit Bloch sphere; $|\chi\rangle_{\mathrm{B}}$ is composed of a linear polarization state and a circular polarization state, which is also a superposition of all four computational basis vectors; and $|\chi\rangle_{\mathrm{C}}$ is a maximally entangled state.

We quantify the quality of our teleportation experiment by looking at the fidelity as defined by

$$
F=\operatorname{Tr}(\hat{\rho}|\chi\rangle\langle\chi|)
$$

where $|\chi\rangle$ is the original state and $\hat{\rho}$ is the density matrix of the 


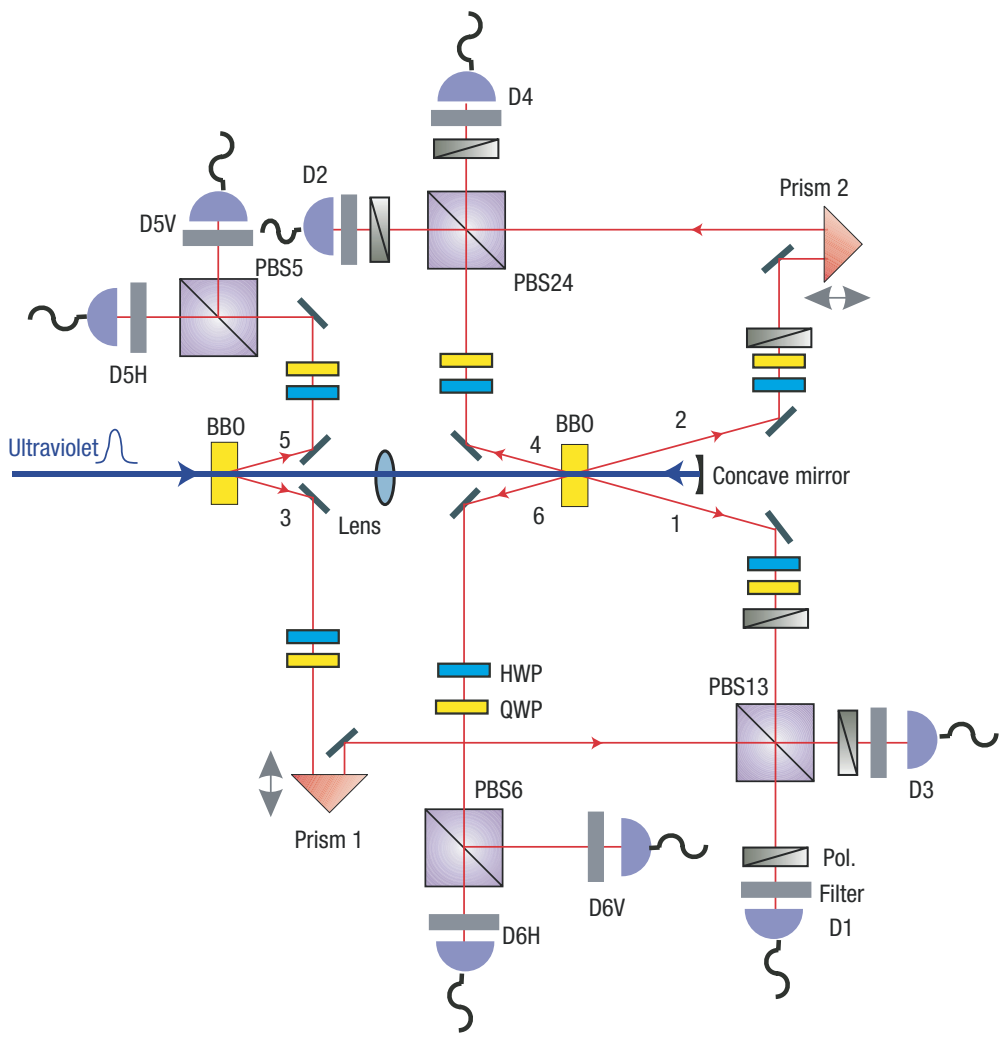

Figure 2 A schematic diagram of the experimental setup. The ultraviolet pulse passes through a BBO crystal to generate a polarization-entangled photon pair in modes 3 and 5 (that is, the first ancillary entangled photon pair). After the first BBO, a 10-cm-focus lens is introduced to refocus the ultraviolet pulse onto the second BBO to produce another entangled photon pair in modes 1 and 2 (to prepare the two qubits to be teleported). Reflected by a concave mirror, the ultraviolet pulse pumps once more into the second BBO and generates the third entangled photon pair in modes 4 and 6 (that is, the second ancillary photon pair). Prisms 1 and 2, both mounted on step motors, are used to compensate the time delay for the interference on polarizing beam splitters PBS13 and PBS24, respectively. PBS5 and PBS6 are used to verify the teleported state with the help of wave plates in front of them. The photons are all detected by silicon avalanched single-photon detectors. Coincidences are recorded with a coincidence unit clocked by the infrared laser pulses. Pol. are linear polarizers and Filter labels the narrow band filter with $\Delta \lambda_{\text {FWHM }}=2.8 \mathrm{~nm}$.

teleported state. To measure the fidelity of two-qubit teleportation, two PBSs (PBS5 and PBS6) and corresponding half-wave and quarter-wave plates (HWP and QWP, respectively), as shown in Fig. 2, are combined properly to analyse the teleported state of photons 5 and 6 .

The fidelity measurements for the $|\chi\rangle_{\mathrm{A}}$ and $|\chi\rangle_{\mathrm{B}}$ teleportation are straightforward. Conditional on detecting a $|+\rangle|+\rangle$ coincidence between D1 and D3, D2 and D4, respectively, we analyse the teleported state of photons 5 and 6 in the $H / V$ basis for the $|\chi\rangle_{\mathrm{A}}$ teleportation; whereas we analyse photon 5 in the \pm basis and photon 6 in the $L / R$ basis for the $|\chi\rangle_{\mathrm{B}}$ teleportation, where $|L\rangle=(|H\rangle+i|V\rangle) / \sqrt{2}$ and $|R\rangle=(|H\rangle-i|V\rangle) / \sqrt{2}$ correspond to the left and right circular polarization states. As the above state analysis only involves orthogonal measurements on individual qubits, the fidelity of the teleported state is directly given by the fraction of observing a $|\chi\rangle_{\mathrm{A}}$ or $|\chi\rangle_{\mathrm{B}}$ state at detectors D5 and D6. The measurement results are shown in Fig. 3. The experimental integration time for each fidelity measurement was about $60 \mathrm{~h}$ and we recorded about 100 desired two-qubit teleportation events. The integration time is slightly longer than would be expected from the original source rate, due to the additional losses at the interference PBSs. On the basis of our original data, we conclude that the fidelity for $|\chi\rangle_{\mathrm{A}}$ or $|\chi\rangle_{\mathrm{B}}$ is $0.86 \pm 0.03$ or $0.75 \pm 0.02$, respectively.

The measurement on the fidelity of the $|\chi\rangle_{C}$ teleportation is a bit more complex, because a complete Bell-state analysis on photons 5 and 6 usually requires nonlinear interaction between them. Fortunately, the fidelity can still be determined by local measurements on individual qubits. To see this, we write the density matrix of $|\chi\rangle_{\mathrm{C}}$ in terms of the Pauli matrices:

$$
|\chi\rangle_{\mathrm{C}}\left\langle\chi|=| \Psi^{-}\right\rangle\left\langle\Psi^{-}\right|=\frac{1}{4}\left(\hat{I}-\hat{\sigma}_{x} \hat{\sigma}_{x}-\hat{\sigma}_{y} \hat{\sigma}_{y}-\hat{\sigma}_{z} \hat{\sigma}_{z}\right) .
$$

By equation (3), we have:

$$
F=\operatorname{Tr}_{\mathrm{C}}\left(\hat{\rho}\left|\Psi^{-}\right\rangle\left\langle\Psi^{-}\right|\right)=\frac{1}{4} \operatorname{Tr}\left[\hat{\rho}\left(\hat{I}-\hat{\sigma}_{x} \hat{\sigma}_{x}-\hat{\sigma}_{y} \hat{\sigma}_{y}-\hat{\sigma}_{z} \hat{\sigma}_{z}\right)\right] .
$$

This implies that we can obtain the fidelity of $|\chi\rangle_{\mathrm{C}}$ teleportation by consecutively carrying out three local measurements $\hat{\sigma}_{x} \hat{\sigma}_{x}, \hat{\sigma}_{y} \hat{\sigma}_{y}$ and $\hat{\sigma}_{z} \hat{\sigma}_{z}$ on the two teleported qubits. The measurement results for the three operators are shown in Fig. 4, each of which took about $60 \mathrm{~h}$. Using equation (4), we determine an experimental fidelity of $0.65 \pm 0.03$.

As can be seen from the above experimental results, all the teleportation fidelities are well beyond the state estimation limit of 0.40 for a two-qubit composite system ${ }^{14}$, hence successfully demonstrating quantum teleportation of a two-qubit composite system. The imperfection of the fidelities is mainly due to the noise caused by emission of two pairs of down-converted photons by a single source ${ }^{6}$. In our experiment, this noise contributes around 10 spurious six-fold coincidences in $60 \mathrm{~h}$ and was not subtracted in the 

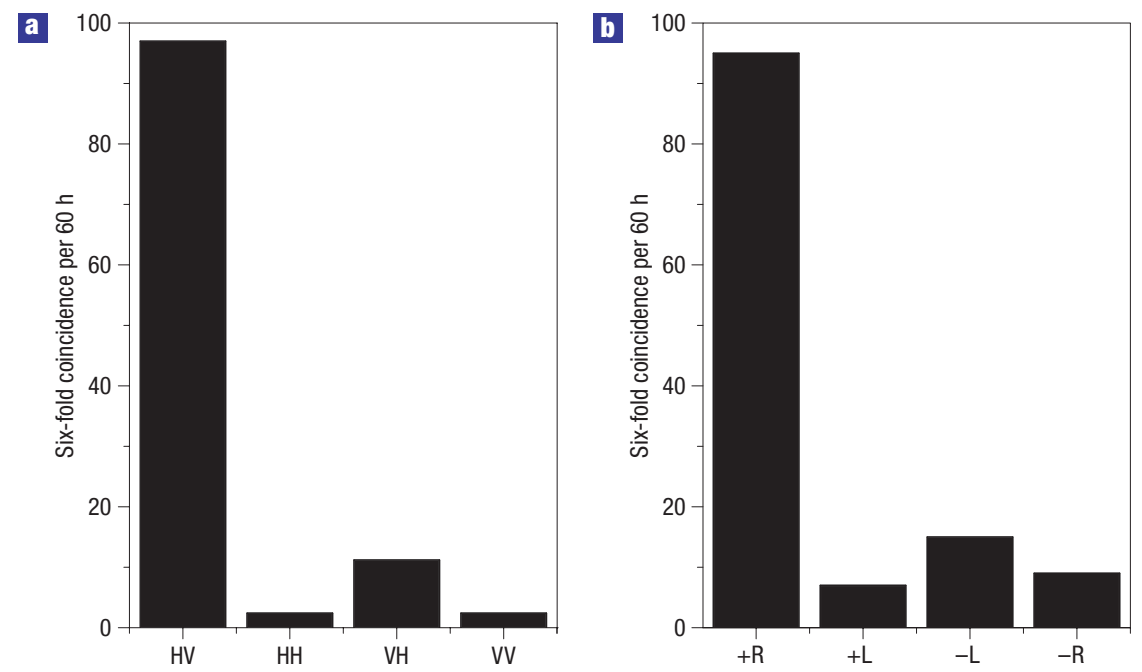

Figure 3 Experimental results for the teleportation of the $|\chi\rangle_{A}$ state and the $|\chi\rangle_{B}$ state. Each measurement takes $60 \mathrm{~h}$. $\mathbf{a}$, The $|\chi\rangle_{A}$ state. We measured photon 5 and 6 in $|H\rangle /|V\rangle$ basis. b. The $|\chi\rangle_{B}$ state. We measured photon 5 in $|+\rangle /|-\rangle$ basis and photon 6 in $|R\rangle /|L\rangle$ basis. The fraction of $|H\rangle|V\rangle(|+\rangle|R\rangle)$ to the sum of all counts shows the fidelity for the teleportation of the $|\chi\rangle_{A}\left(|\chi\rangle_{B}\right)$ state in $\mathbf{a}(\mathbf{b})$.
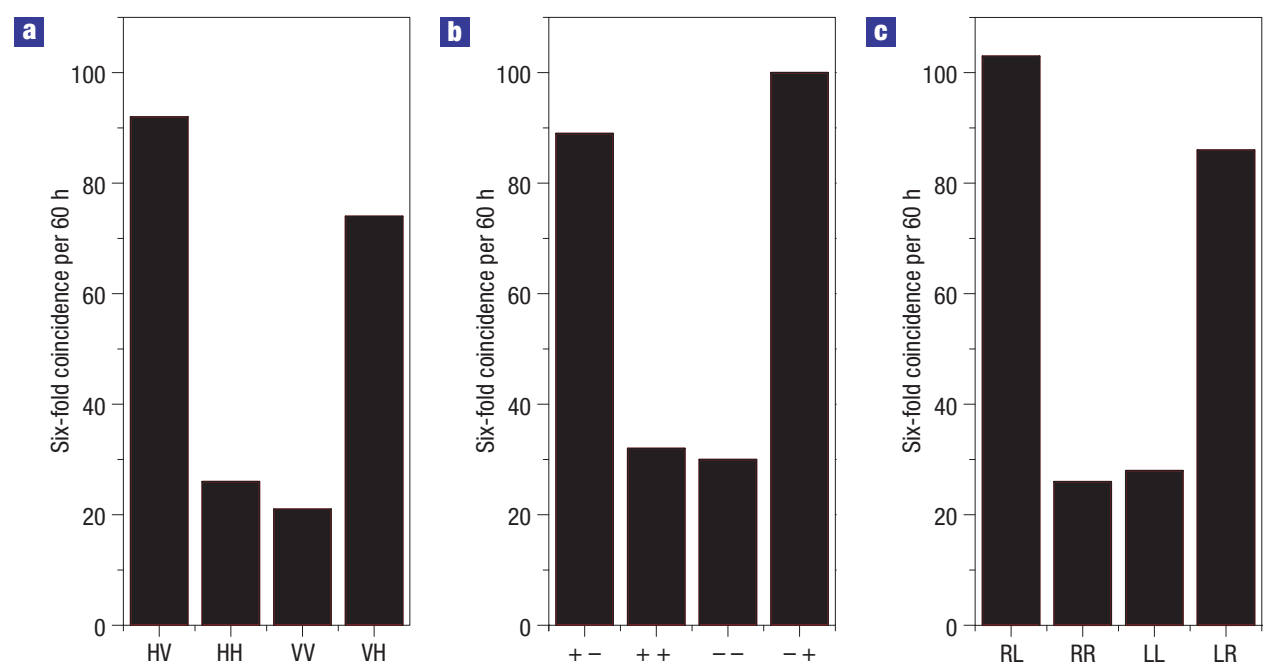

Figure 4 Experimental results for $|\chi\rangle_{\mathbf{c}}$ teleportation. Three complementary bases were used: $\mathbf{a},|H\rangle /|V\rangle, \mathbf{b},|+\rangle /|-\rangle$ and $\mathbf{c},|R\rangle /|L\rangle$ corresponding to the three different local measurements $\hat{\sigma}_{x} \hat{\sigma}_{x}, \hat{\sigma}_{y} \hat{\sigma}_{y}$ and $\hat{\sigma}_{z} \hat{\sigma}_{z}$. Each measurement takes $60 \mathrm{~h}$. In a, whenever there is $\mathrm{a}|H\rangle|H\rangle$ or $|V\rangle|V\rangle$ coincidence, the result of the $\hat{\sigma}_{x} \hat{\sigma}_{x}$ is +1 , whereas $|H\rangle|V\rangle$ or $|V\rangle|H\rangle$ represents -1 . In $\mathbf{b},|+\rangle|+\rangle$ or $|-\rangle|-\rangle$ represents +1 , whereas $|+\rangle|-\rangle$ or $|-\rangle|+\rangle$ represents -1 . In $\mathbf{c},|R\rangle|R\rangle$ or $|L\rangle|L\rangle$ displaces +1 , whereas $|R\rangle|L\rangle$ or $|L\rangle|R\rangle$ displaces -1 .

fidelity estimation. Besides that, the limited interference visibility and imperfect entangled state also reduce our teleported fidelities. We notice that the fidelities of $|\chi\rangle_{B}$ and $|\chi\rangle_{C}$ teleportation are worse than those of $|\chi\rangle_{\mathrm{A}}$. This is because the fidelities of $|\chi\rangle_{\mathrm{B}}$ and $|\chi\rangle_{\mathrm{C}}$ teleportation depend on the interference visibility on PBS13 and PBS24. Moreover, as the quality of the initial entangled state $\left(|\chi\rangle_{\mathrm{C}}\right)$ is not as good as for the disentangled states $\left(|\chi\rangle_{\mathrm{A}}\right.$ and $\left.|\chi\rangle_{\mathrm{B}}\right)$, the fidelity of $|\chi\rangle_{C}$ teleportation is worse than that of the other two.

In summary, we have developed and exploited a six-photon interferometer to report the first experimental teleportation of a two-qubit composite system. Not only does our experiment present an important step towards teleportation of a complex system, the techniques developed also enable future experimental investigations on novel quantum communication and computation protocols. For example, using a slightly modified experimental setup, photons 3, 4, 5 and 6 can first be prepared in a four-photon cluster state ${ }^{26}$, which can be further exploited to demonstrate teleportation-based controlled-NOT operation between photons 1 and 2; this is the kernel of fault-tolerant quantum computation ${ }^{3}$. In addition, whereas a modified six-photon interferometer can be used to prepare a six star-ring cluster state ${ }^{27}$ and further implement universal quantum error-correction code ${ }^{16}$ by carrying out a projection measurement on the input qubit, our six-photon interferometer can also be modified to produce various four-, five- 
and six-photon clusters ${ }^{28}$, which are essential resources for one-way quantum computation ${ }^{17,18}$.

Received 13 July 2006; accepted 25 August 2006; published 17 September 2006.

\section{References}

1. Bennett, C. H. et al. Teleporting an unknown quantum state via dual classical and Einstein-Podolsky-Rosen channels. Phys. Rev. Lett. 70, 1895-1899 (1993).

2. Briegel, H.-J., Dür, W., Cirac, J. I. \& Zoller, P. Quantum repeaters: The role of imperfect local operations in quantum communication. Phys. Rev. Lett. 81, 5932-5935 (1998).

3. Gottesman, D. \& Chuang, I. L. Demonstrating the viability of universal quantum computation using teleportation and single-qubit operations. Nature 402, 390-393 (1999).

4. Knill, E., Laflamme, R. \& Milburn, G. J. A scheme for efficient quantum computation with linear optics. Nature 409, 46-52 (2001).

5. Grover, L. K. Quantum telecomputation. Preprint at $<$ http://arxiv.org/abs/quant-ph/9704012> (1997).

6. Bouwmeester, D. et al. Experimental quantum teleportation. Nature 390, 575-579 (1997).

7. Boschi, D., Branca, S., De Martini, F., Hardy, L. \& Popescu, S. Experimental realization of teleporting an unknown pure quantum state via dual classical and Einstein-Podolsky-Rosen channels. Phys. Rev. Lett. 80, 1121-1125 (1998).

8. Pan, J.-W., Bouwmeester, D., Weinfurter, H. \& Zeilinger, A. Experimental entanglement swapping: entangling photons that never interacted. Phys. Rev. Lett. 80, 3891-3894 (1998).

9. Marcikic, I., de Riedmatten, H., Tittel, W., Zbinden, H. \& Gisin, N. Long-distance teleportation of qubits at telecommunication wavelengths. Nature 421, 509-513 (2003).

10. Ursin, R. et al. Quantum teleportation across the Danube. Nature 430, 849 (2004)

11. Zhao, Z. et al. Experimental demonstration of five-photon entanglement and open-destination teleportation. Nature 430, 54-58 (2004).

12. Riebe, M. et al. Deterministic quantum teleportation with atoms. Nature 429, 734-737 (2004).

13. Barrett, M. D. et al. Deterministic quantum teleportation of atomic qubits. Nature $\mathbf{4 2 9}$, 737-739 (2004).

14. Hayashi, A., Hashimoto, T. \& Horibe, M. Reexamination of optimal quantum state estimation of pure states. Phys. Rev. A 72, 032325 (2005).

15. Jacobs, B. C., Pittman, T. B. \& Franson, J. D. Quantum relays and noise suppression using linear optics. Phys. Rev. A 66, 052307 (2002).
16. Calderbank, A. R. \& Shor, P. W. Good quantum error-correcting codes exist. Phys. Rev. A 54, 1098-1105 (1996)

17. Raussendorf, R. \& Briegel, H. J. A one-way quantum computer. Phys. Rev. Lett. 86, 5188-5191 (2001).

18. Walther, P. et al. Experimental one-way quantum computing. Nature 434, 169-176 (2005).

19. Lee, J. \& Kim, M. S. Entanglement teleportation via Werner states. Phys. Rev. Lett. 84, $4236-4239$ (2000)

20. Rigolin, G. Quantum teleportation of an arbitrary two-qubit state and its relation to multipartite entanglement. Phys. Rev. A 71, 032303 (2005).

21. Cleve, R., Gottesman, D. \& Lo, H.-K. How to share a quantum secret. Phys. Rev. Lett. 83, 648-651 (1999).

22. Kwiat, P. G. et al. New high intensity source of polarization-entangled photon pairs. Phys. Rev. Lett. 75, 4337-4341 (1995).

23. Pan, J.-W., Daniell, M., Gasparoni, S., Weihs, G. \& Zeilinger, A. Experimental demonstration of four-photon entanglement and high-fidelity teleportation. Phys. Rev. Lett. 86, 4435-4438 (2001).

24. Zukowski, M., Zeilinger, A. \& Weinfurter, H. Entangling photons radiated by independent pulsed source. Ann. NY Acad. Sci. 755, 91-102 (1995).

25. Pan, J.-W. \& Zeilinger, A. Greenberger-Horne-Zeilinger-state analyzer. Phys. Rev. A 57, 2208-2211 (1998)

26. Zou, X.-B. \& Mathis, W. Generating a four-photon polarization-entangled cluster state. Phys. Rev. A 71, 032308 (2005).

27. Hein, M., Eisert, J. \& Briegel, J. Multiparty entanglement in graph states. Phys. Rev. A 69, 062311 (2004).

28. Browne, D. E. \& Rudolph, T. Resource-efficient linear optical quantum computation. Phys. Rev. Lett. 95, 010501 (2005).

\section{Acknowledgements}

This work was supported by the Marie Curie Excellence Grant of the EU and the Alexander von

Humboldt Foundation. This work was also supported by the National Natural Science Foundation of China and the Chinese Academy of Sciences.

Correspondence and requests for materials should be addressed to Q.Z. or J.-W.P.

Supplementary Information accompanies this paper on www.nature.com/naturephysics.

Competing financial interests

The authors declare that they have no competing financial interests.

Reprints and permission information is available online at http://npg.nature.com/reprintsandpermissions/ 